\title{
PP06
}

\section{QUANTIFYING PERCEIVED CHROMA CHANGES BY HUNT EFFECT IN LIGHTING}

\author{
Yuki Kawashima et al.
}

DOI 10.25039/x46.2019.PP06

from

CIE x046:2019

\section{Proceedings}

of the

29th CIE SESSION

Washington D.C., USA, June 14 - 22, 2019

(DOI 10.25039/x46.2019)

The paper has been presented at the 29th CIE Session, Washington D.C., USA, June 14-22, 2019. It has not been peer-reviewed by CIE.

(C) CIE 2019

All rights reserved. Unless otherwise specified, no part of this publication may be reproduced or utilized in any form or by any means, electronic or mechanical, including photocopying and microfilm, without permission in writing from CIE Central Bureau at the address below. Any mention of organizations or products does not imply endorsement by the CIE.

This paper is made available open access for individual use. However, in all other cases all rights are reserved unless explicit permission is sought from and given by the CIE.

CIE Central Bureau

Babenbergerstrasse 9

A-1010 Vienna

Austria

Tel.: +4317143187

e-mail: ciecb@cie.co.at

www.cie.co.at 


\title{
QUANTIFYING PERCEIVED CHROMA CHANGES BY HUNT EFFECT IN LIGHTING
}

\author{
Kawashima, Y., Ohno, Y. \\ National Institute of Standards and Technology, Gaithersburg, MD USA \\ yuki.kawashima@nist.gov
}

DOI 10.25039/x46.2019.PP06

\begin{abstract}
Our previous experiment showed that the Hunt Effect was effective at normal indoor lighting levels. The purpose of this study is to quantify the level of perceived chroma increase by the Hunt Effect. Two identical colour patches were placed on the left and the right side of the booth. The left side of the booth was illuminated at $1000 \mathrm{~lx}$ with a broadband spectrum and the right side was illuminated at $100 \mathrm{~lx}$ or $300 \mathrm{~lx}$ with chroma-increasing lights with different chroma increase levels. Subjects viewed each side of the booth with each eye (haploscopic viewing condition) and were asked to select a light of the right side that matched the colour of the rightside target closest to the left one. Results showed that increased chroma by 15 to 18 in $\Delta C^{*}$ ab were needed for the right side to match perceived chroma. The chroma increase was smaller at $300 \mathrm{~lx}$.
\end{abstract}

Keywords: Hunt Effect, colour fidelity, chroma, saturation, natural object, lighting

\section{Introduction}

The Hunt Effect is a perception effect, by which perceived chroma of object colours appear less saturated at low light levels than at high light levels (Hunt, 1950). This means that in order to make the appearance of the objects at the low light levels close to that at high light levels, the chroma saturation level of the lighting needs to be increased. If the Hunt Effect is effective at normal indoor lighting levels, it is considered that objects in indoor lighting (relatively low illuminance levels) would appear less saturated than those at outside daylight (much higher illuminance levels).

Chroma saturation is related to the colour preference and memory. It was shown that the preferred colours for natural objects are more saturated than the actual colours of those (Sanders, 1959; Judd, 1967) and that the colours for the natural objects are memorized with higher saturation than those actual colours (Newhall et al., 1957; Bartleson, 1960). However, if the appearance of objects at daylight is considered as the reference for the appearance, the Hunt Effect may relate to not only preference or memory but also colour fidelity of lighting. Therefore, it is considered that increasing the chroma of object by a light source in indoor lighting can bring a sort of higher colour fidelity.

CRI (CIE, 1995) and Colour fidelity index (IES, 2015; CIE, 2017) are known as metrics for the colour fidelity. Especially, the CRI is defined by International Commission on Illumination (CIE), and is known and used as the only internationally accepted metric to evaluate the colour rendering performance of lighting products. In the calculation of the CRI values, colour differences of 14 Munsell samples, between under a reference illuminant (a blackbody or daylight illuminant) and under a test light, are calculated. Regardless of increasing or decreasing in the chroma saturation, colour shifts by a test light in any directions are equally calculated. Such colour shifts reduce the CRI values. Since colour shifts in any direction are equally penalized in the CRI, an increase of chroma to compensate the Hunt Effect would also penalize the CRI. Therefore, it is important to verify and quantify the degree in which the Hunt Effect affects the perception of colour saturation and evaluate impact on colour fidelity.

Our previous experiment showed that the Hunt Effect is effective at normal indoor lighting levels, tested at $100 \mathrm{Ix}$ and $1000 \mathrm{Ix}$, and the perceived chroma decrease at $100 \mathrm{~lx}$ from the $1000 \mathrm{Ix}$ level was estimated to be approximately 1 to 10 in $\Delta C^{*}$ ab. Some studies also showed that the 
illuminance level of lightings affect the preference for the objects, suggesting this could be due to the Hunt Effect (Islam et al., 2013; Wei et al., 2018). However, it is still not clear how much perceived chroma shifts occur for each colour of objects by Hunt Effect.

The purpose of this study, therefore, is to quantify the perceived chroma shifts by the Hunt Effect. A vision experiment was conducted for saturation matching of coloured objects under different illuminance levels using a spectrally-tuneable lighting double booth and with a haploscopic viewing condition.

\section{Methods}

A saturation matching experiment was conducted using a spectrally-tuneable lighting double booth. Two identical colour patches of saturated red or green colour were placed on the right and left side of the booth and illuminated by a reference light at $1000 \mathrm{~lx}$ on one side and matching lights on the other side at $100 \mathrm{~lx}$ or $300 \mathrm{~lx}$. The chroma saturation of the right-side patch was changed by using chroma-enhancing light spectra at several different levels. The subject was adapted in haploscopic view condition, so his/her left eye was adapted to the left booth at $1000 \mathrm{Ix}$ and the right eye to the right booth at $100 \mathrm{~lx}$ illumination. As the saturation level changed on the right-side sample, the subject was asked to choose a matching light that made the chroma saturation of the right-side sample closest to the left one.

Since each eye will not be perfectly adapted to very different light levels with the haploscopic view, it was tested how completely the subject adapted to each light using grey patches before starting the saturation matching. After the subject was fully adapted to $1000 \mathrm{~lx}$ and $100 \mathrm{Ix}$ for their left and right eyes, a reference grey patch was placed on the left-side booth and matching grey patches having several different lightness levels were placed on the right-side booth. The subject was asked to choose a grey patch that matched the brightness of the left-side one (the reference grey patch). If each eye was completely adapted to each light, the matching grey patch with same grey level as that of reference grey patch should be chosen. The difference between the grey level of the matching patch and the reference grey patch represents incompleteness of the adaptation, which was used to correct the experiment results.

\subsection{Apparatus}

The experiment was conducted in a dark room using a double-lighting booth with spectrally tuneable light sources shown in Figure 1. The double-lighting booth consists of a viewing compartment and a light source compartment and is divided into left and right by a partition wall at its centre. The light source compartment was hidden by a top cover during the experiment and the subject did not see the light source directly. A view divider is attached to the centre partition wall for haploscopic view. The inside of viewing compartment and the centre partition wall are painted in grey. The size of viewing area on each side is $50 \mathrm{~cm}$ wide and $37 \mathrm{~cm}$ high, and $65 \mathrm{~cm}$ deep. Each side of light source compartment is equipped with a spectrally tuneable light source which has 16 channels of LED spectra (ranged from $495 \mathrm{~nm}$ to $730 \mathrm{~nm}$ peak). The light sources are controlled with a computer program that allows spectrum settings by changing intensity for each channel. The light-emitting surface of the light source is a diffuser with a $10 \mathrm{~cm}$ diameter, and there is a large light-transmitting diffuser between the light source compartment and viewing compartment in the booth, with which good spatial colour uniformity is provided in the viewing area.

The light sources need a long time (several hours) to reach sufficient stability required for this experiment after turning on. Therefore, the light sources were set to initial condition of the experiment ( $1000 \mathrm{~lx}$ for the left, $100 \mathrm{~lx}$ for the right, both CCTs were $3000 \mathrm{~K}$ or $5000 \mathrm{~K}$ ) at least 2 hours prior to starting the experiment. The spectral distributions were measured at the centre of the table top surface in the cubicle with an array spectrometer on all light settings before and after the experiment every day. It was ensured that the measured chromaticity values were within 0.0005 in u' and v' from the target values.

The expanded uncertainties $(k=2)$ of the measurements were estimated to be 0.0012 in $u$ ', 0.0011 in $v^{\prime}, 0.0009$ in Duv, $15 \mathrm{~K}$ in CCT at $3000 \mathrm{~K}$ and $40 \mathrm{~K}$ at $5000 \mathrm{~K}$ for the light spectra used in this experiment. The expanded uncertainty $(k=2)$ in the relative chromaticity measurement between each pair of light was 0.0002 in $u^{\prime}$ and $v^{\prime}$, which is the typical repeatability of the instrument. The expanded uncertainty of illuminance of the 
spectroradiometer is estimated $3 \%(k=2)$ for directional incident light, and its uncertainty for relative measurement was $0.2 \%$, which is the typical reproducibility of the instrument.
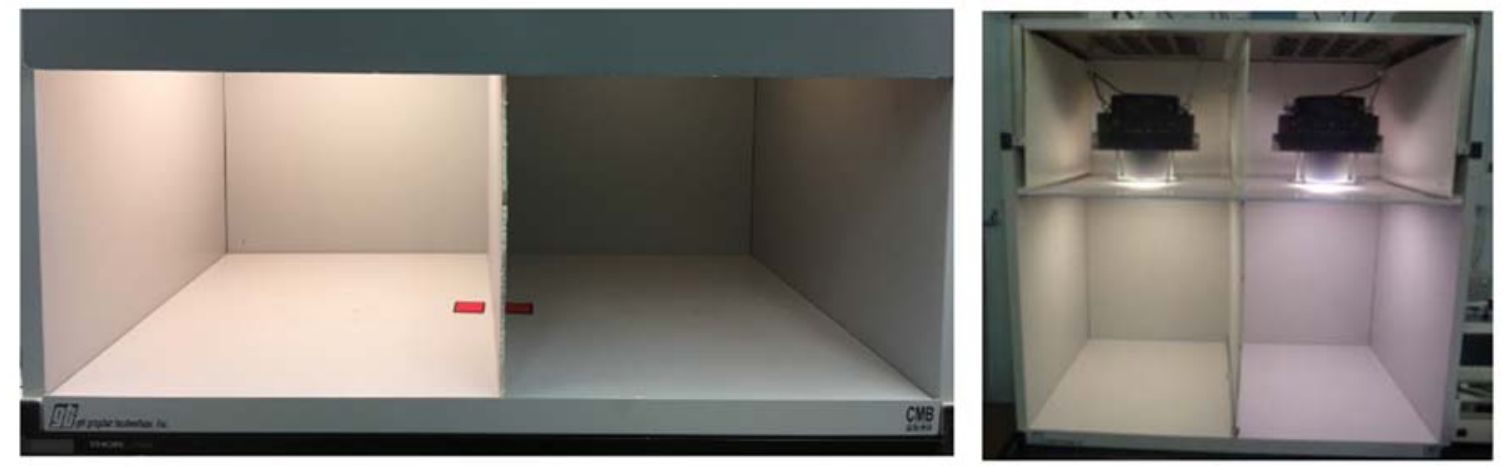

Figure 1 - Photograph of the double booth used for the experiment. The photos show when the top cover is placed during experiment (left) and when it is removed (right).

\subsection{Colour patches and light sources}

Seven test grey patches $(4 \mathrm{~cm} \times 4 \mathrm{~cm})$ were used for grey scale matching. The surfaces of these test patches were plain paper printed with $50 \%, 53 \%, 56 \%, 59 \%, 62 \%$ and $65 \%$ grey (luminous reflectance for Illuminant A). The reference grey patch placed on the left side of the booth was a $50 \%$ grey patch, identical to the $50 \%$ grey test patch. The six grey patches $(50 \%$ to $65 \%$ grey) were used as the matching grey patches on the right side.

Two pairs of identical colour patch, red and green, were used as targets for the chroma saturation matching. These patches were cut out from the Macbeth Colour Checker Chart ${ }^{1}$, and were No.15 patches and No.14 patches of the chart respectively $(5 \mathrm{~cm} \times 5 \mathrm{~cm}$, in Munsell

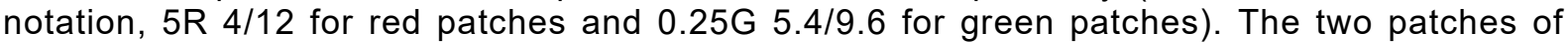
identical colour were placed on the right and left-side booth respectively as shown in Figure 1. Each patch was at near the centre partition wall and at $30 \mathrm{~cm}$ away from the front of the booth. The subject observed the patches at a viewing angle of approximately $45^{\circ}$ from the normal to the patch surface, and the viewing distance between the targets and subjects was approximately $55 \mathrm{~cm}$. The spectral reflectance of patches were measured using a telephoto type spectroradiometer prior to the experiment.

A reference light at $1000 \mathrm{~lx}$ was presented in the left-side booth, and the matching lights at $100 \mathrm{Ix}$ or $300 \mathrm{Ix}$ were presented in the right-side booth. The reference and matching lights were set at same CCT, $3000 \mathrm{~K}$ or $5000 \mathrm{~K}$, with Duv $=0$. At the CCT $5000 \mathrm{~K}$ condition, only the matching lights with $100 \mathrm{Ix}$ were used in the experiment. A set of one reference light and seven or six matching lights were made for each colour patch at each CCT. Figure 2 shows the $a^{*}, b^{*}$ coordinates for the patches with the reference and matching lights on CIELAB colour space. When preparing the matching lights for different chroma increase levels, it is important that only chroma changes without changing hue angle. Such settings were made possible by the spectral tuneable sources of the booth, and the plots in Figure $\mathbf{2}$ show that it was fairly successfully done.

1 Certain commercial products are identified in this paper for information. Such identification does not imply recommendation or endorsement by the National Institute of Standards and Technology, nor does it imply that the materials or equipment identified are necessarily the best available for the purpose. 
$3000 \mathrm{~K}$, Matching lights at $100 \mathrm{Ix}$

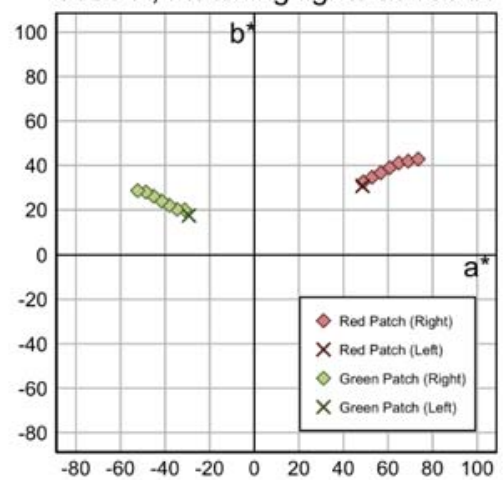

$3000 \mathrm{~K}$, Matching lights at $300 \mathrm{Ix}$

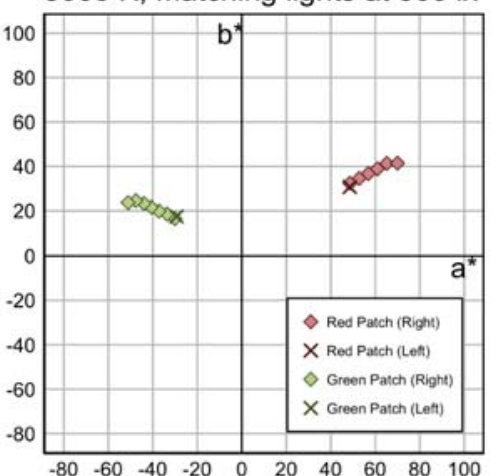

$5000 \mathrm{~K}$, Matching lights at $100 \mathrm{~lx}$

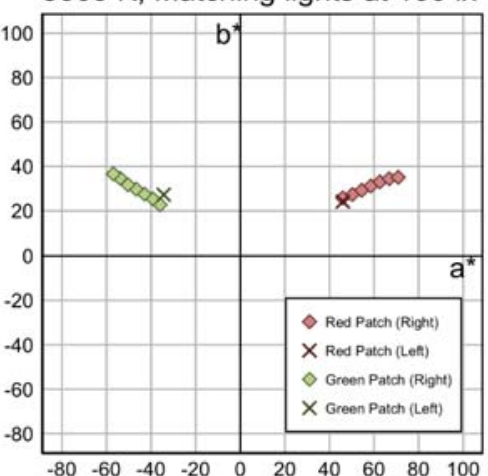

Figure 2 - Colour coordinates for the patches on CIELAB. CCT and illuminance levels of the matching lights are $3000 \mathrm{~K} / 100 \mathrm{~lx}$ (left), $3000 \mathrm{~K} / 300 \mathrm{~lx}$ (centre), and $5000 \mathrm{~K} / 100 \mathrm{~lx}$ (right). X marks show patches under the reference light and diamond marks show patches under the matching lights.

In each light set, the reference light was made so that the left-side patch has approximately the same $a^{*}, b^{*}$ coordinate as that for the right-side patch with the matching light at the lowest saturation. Thus, the matching light at the lowest chroma saturation was the same colour as the reference light on the left side. The right-side patch has increased chroma saturations with the chroma-enhancing matching light by up to $\Delta C^{*}{ }_{\mathrm{ab}} \approx 28$ for the red patch and $\Delta C^{*}{ }_{\mathrm{ab}} \approx 26$ increase for the green patch from the chroma at the lowest (neutral) saturation level. Figure 3 shows the spectra of the reference lights and these were broadband spectra. The spectra for the matching lights are shown in Figure 4, and the matching lights were set to RGBA spectra.
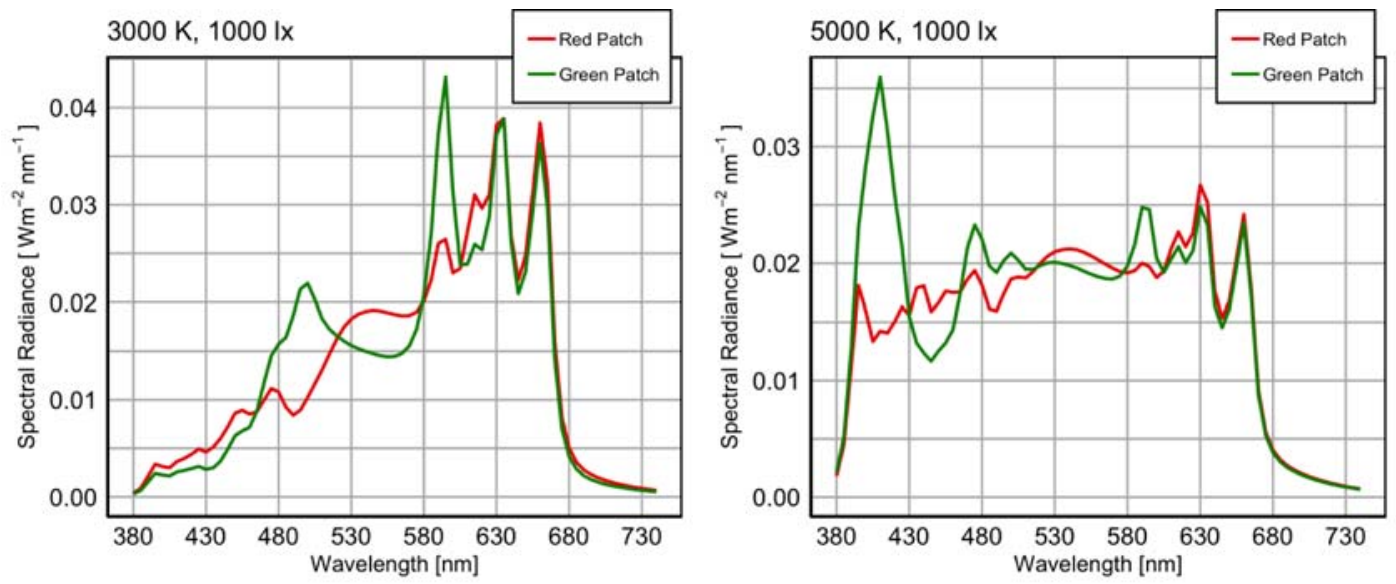

Figure 3 - The spectral distributions of the reference lights for each patch at $3000 \mathrm{~K}$ (left) and $5000 \mathrm{~K}$ (right). 
3000 K, 100 Ix, for Red Patch

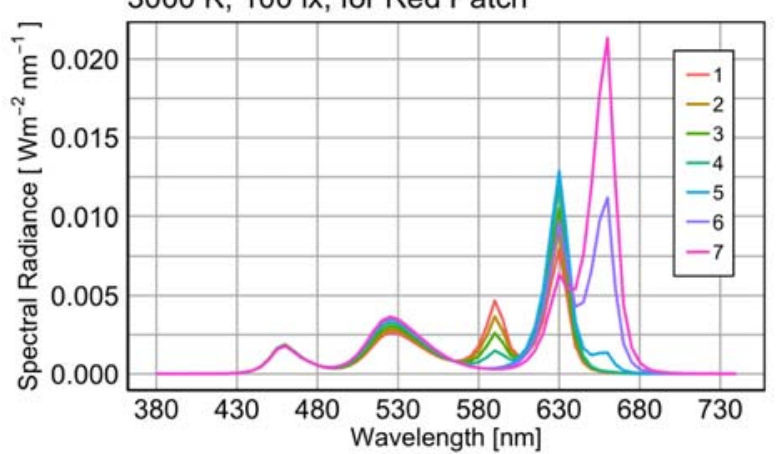

3000 K, 300 Ix, for Red Patch

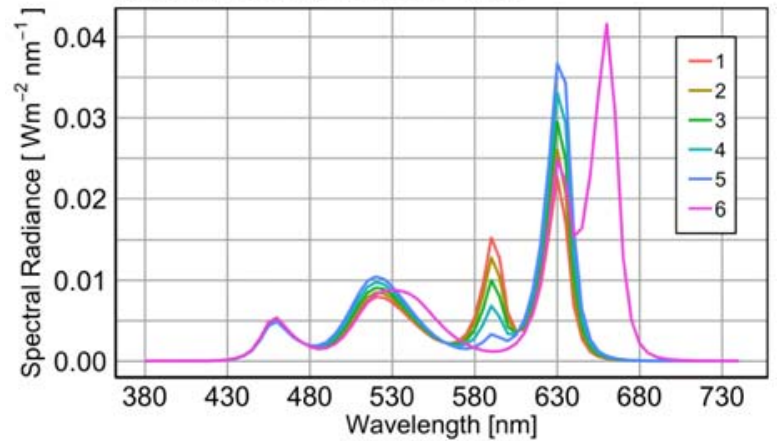

5000 K, 100 Ix, for Red Patch

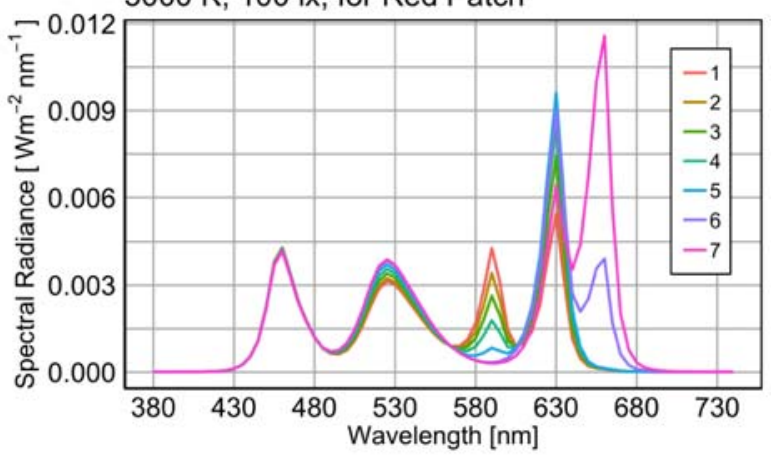

3000 K, 100 Ix, for Green Patch
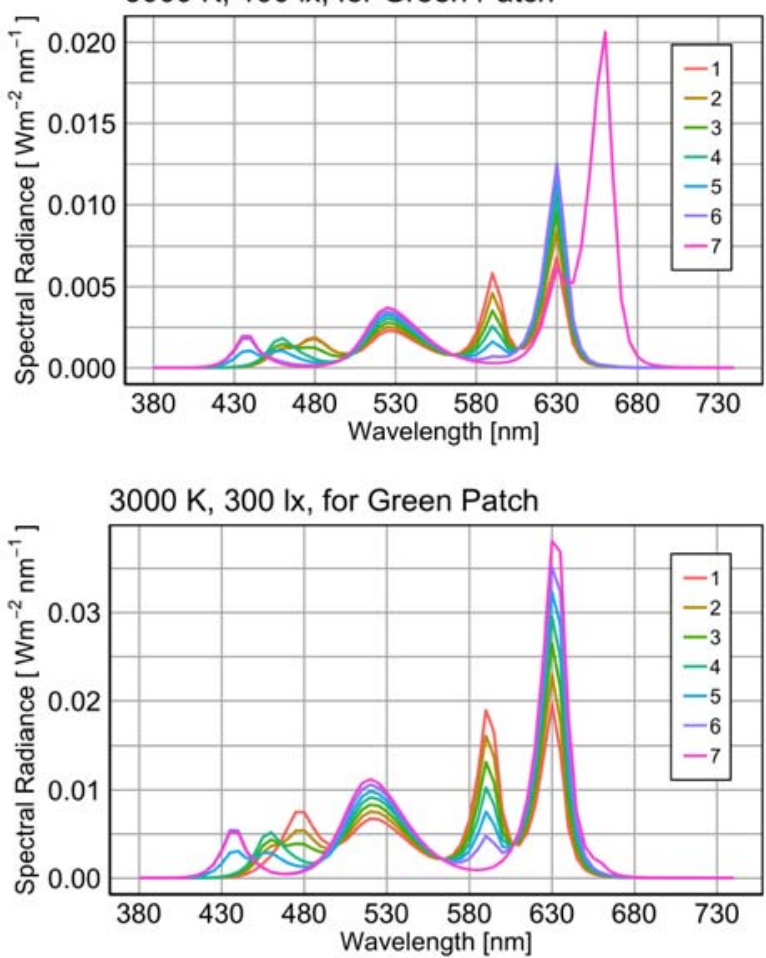

5000 K, 100 Ix, for Green Patch

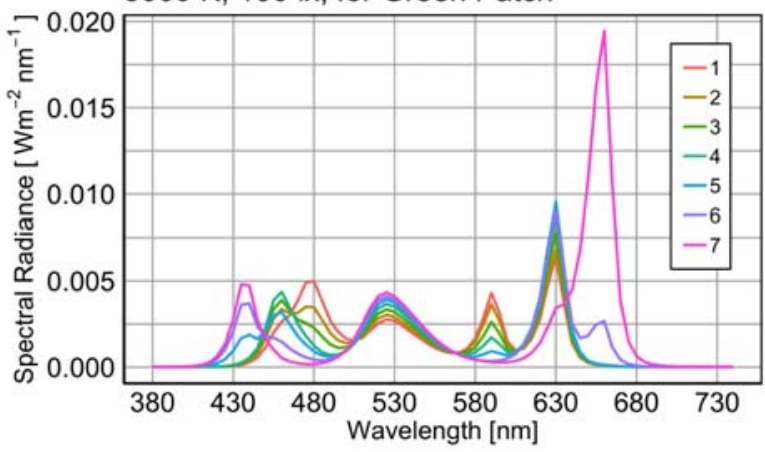

Figure 4 - The spectral distributions of the test lights for each patch at each CCT/illuminance condition. Each line shows the chroma saturation level.

\subsection{Procedures}

Each subject was tested for normal colour vision using Ishihara Test book before starting the experiment. The subject was instructed to be seated in front of the booth and observed the booth with his/her forehead and nose placed against the view divider (haploscopic view) as shown in Figure 5. With the haploscopic view, the left eye viewed only the left-side booth $(1000 \mathrm{~lx})$, and the right eye viewed only the right-side both $(100 \mathrm{Ix})$. 


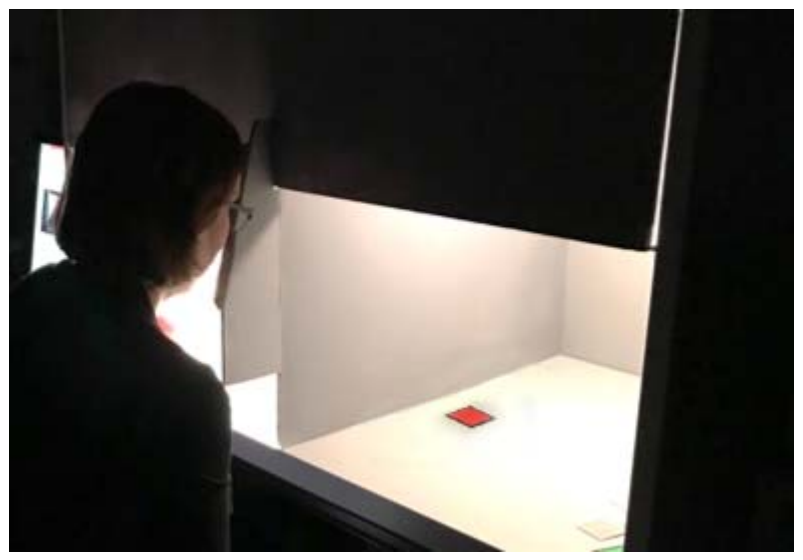

Figure 5 - Experimental scene of the Haploscopic view

The saturation matching experiment consisted of three sessions according to the CCT condition and the illuminance of the matching lights. In the first and second session, the matching lights with $100 \mathrm{Ix}$ were used and CCT for each session was at $3000 \mathrm{~K}$ and $5000 \mathrm{~K}$, or $5000 \mathrm{~K}$ and $3000 \mathrm{~K}$. The order of CCT condition was randomly assigned for each subject. In the third session, the matching lights with $300 \mathrm{Ix}$ were used and CCT was at only $3000 \mathrm{~K}$. For each session, the saturation matching was conducted with the light set for red patches first, followed by that for the green patches.

Before starting each session, the reference and matching light with the lowest saturation was presented on the left and right-side booth respectively. The subject was adapted to each light for each eye for three minutes. An instruction for the experiment was given to subjects during the adaptation time. After the three minutes adaptation, the grey patches were placed, and the subject conducted the grey scale matching. The results of this grey matching were used to correct the experimental results for imperfect adaptation with the haploscopic view.

After finishing the grey scale matching, grey patches were removed and the colour patches were put on the booth. Then the subject conducted the saturation matching. The matching lights were presented in the order from the lowest saturation to highest saturation. Each saturation level was shown only about one second each and moved to next saturation level, asking the subject to make judgment quickly, because it was noticed that the colour of the strong red or green sample on the $100 \mathrm{Ix}$ side gradually appearing less and less saturated if viewed for longer time, presumably due to local colour adaptation. After the matching light with the maximum saturation, the first matching light was presented. Presentation of such matching lights was repeated two or three times if requested by the subject.

\subsection{Observers}

A total of 22 subjects participated in this experiment. All subjects have normal colour vision as tested using Ishihara Test book. They were 9 males and 13 females with their ages from 18 to 63, consisting of 16 Caucasians, 4 Asians and 2 African Americans. They were summerinternship students or NIST employees, who were not experts on colour or lighting and completely naïve as to the purpose of the experiment.

\section{Results}

In the grey scale matching, typically $53 \%$ to $56 \%$ of the matching grey patches were selected by the subjects, indicating significant individual variations. The lightness $L^{*}$ increase from the $50 \%$ grey patch was calculated for each selected matching grey patch. In order to correct the chroma saturation of the colour patch based on the grey scale matching, this $L^{*}$ increase was used. First, chroma saturation increase of the colour patch was calculated as a function of the $L^{*}$ increase by multiplying the spectral reflectance. Then, the chroma saturation increase for the $L^{*}$ increase of the selected matching grey patch was used as a correction value of the chroma saturation for the colour patch. 
Chroma differences between the colour patch with the selected matching light on the right side and the colour patch with the reference light on the left side was calculated for each colour (red and green) and CCT/illuminance condition. These chroma differences were normalized by the chroma of the colour patch with the reference light on the left side for each colour and CCT, and expressed as relative change in \% of chroma, since the chroma of those colour patches were different for each colour and CCT. Figure 6 shows the mean chroma increase of the colour patch with selected matching light on the right side for each colour and CCT at $100 \mathrm{Ix}$, corrected with the grey matching results. For the red patch, the mean matching chroma were $25.8 \%$ and $29.7 \%$ higher than the chroma for the left-side patch at $3000 \mathrm{~K}$ and $5000 \mathrm{~K}$ respectively. For the green patch, the mean matching chroma saturations were $55.0 \%$ and $41.1 \%$ higher than the left-side patch at $3000 \mathrm{~K}$ and $5000 \mathrm{~K}$ respectively. Moreover, there were statistically significant differences for the mean matching chroma increase between the red patch and the green patch (paired t-test, $t(21)=-10.7, p<0.001$ for $3000 \mathrm{~K}, t(21)=-6.47, p<0.001$ for $5000 \mathrm{~K})$. The green patch needed more chroma saturation to match that of the left side.
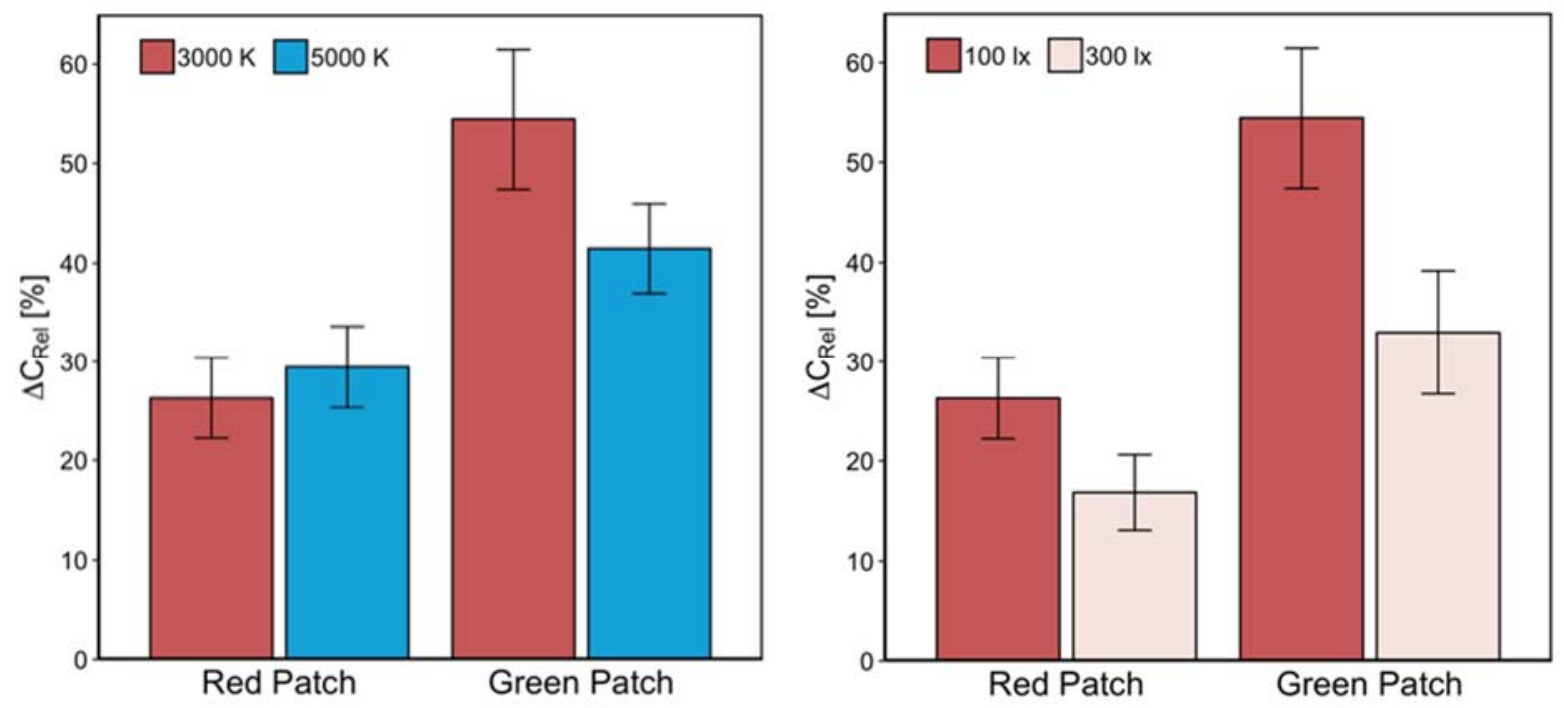

Figure 6 - Chroma increases of the right-side colour patch, for $3000 \mathrm{~K}$ and $5000 \mathrm{~K}$ at $100 \mathrm{Ix}$ (left) and for $3000 \mathrm{~K}$ at $100 \mathrm{~lx}$ and $300 \mathrm{~lx}$ (right). Each bar shows the mean chroma increase. Error bar shows the Standard Error.

As comparison between the red and green patches at $100 \mathrm{Ix}$, the green patch needed more chroma saturation to match that of the left side at $300 \mathrm{~lx}$. It was found that the statistically significant difference for the mean matching chroma increase between with the red and green patch at $300 \mathrm{Ix}$ (paired t-test, $t(21)=-7.10, p<0.001$ ). The mean matching chroma saturations at $300 \mathrm{Ix}$ were smaller than those at $100 \mathrm{~lx}$ for each colour patch. There were statistically significant differences for the mean matching chroma increase between $100 \mathrm{~lx}$ and $300 \mathrm{Ix}$ for each colour patch (paired t-test, $t(21)=5.82, p<0.001$ for the Red patch, $t(21)=6.43$, $p<0.001$ for the Green patch).

\section{Conclusions}

The changes in perceived chroma due to the Hunt Effect have been quantified for the red and green patches between $100 \mathrm{Ix}$ and $1000 \mathrm{Ix}$. The experiment results showed that, at $100 \mathrm{Ix}$, the perceived chroma of the red patch was shown to decrease by around $30 \%$ and the that of the green patch was shown to decrease by around $50 \%$ compared to those at $1000 \mathrm{Ix}$. The large changes of perceived chroma for the green colour compared to red colour indicates that colour vision is more sensitive to red. The chroma increase values indicated above might have been overestimated due to the local chromatic adaptation effects. Further experiments are needed to investigate this effect and to provide more accurate evaluation of perceived chroma decrease at low light levels. Also, this experiment was limited to evaluation of only red and green samples due the capability of light sources. Experiments using other methods are planned to evaluate the Hunt Effect for other colours. 


\section{Acknowledgement}

The authors thank Semin Oh at Ulsan National Institute of Science and Technology, South Korea, for his valuable contributions in preparing and assisting the vision experiment at NIST. The experiment using human subjects in this study was conducted under NIST Institutional Review Board approval PML-16-0001.

\section{References}

BARTLESON, C.J. 1960. Memory Colours of Familiar Objects*. Journal of the Optical Society of America, 50(1), 73-77. (DOI: 10.1364/JOSA.50.000073)

CIE 1995. CIE 013.3-1995. Method of Measuring and Specifying Colour Rendering Properties of Light Sources.

CIE 2017. CIE 224:2017. CIE 2017 COLOUR FIDELITY INDEX FOR ACCURATE SCIENTIFIC USE.

HUNT, R.W.G. 1950. The Effects of Daylight and Tungsten Light-Adaptation on Colour Perception. Journal of the Optical Society of America, 40(6), 362-371. (DOI: 10.1364/JOSA.40.000362)

IES 2015. TM-30-15. IES Method for Evaluating Light Source Colour Renditon. New York, NY: The Illuminating Engineering Society of North America.

ISLAM, M. et al. 2013. User preferences for LED lighting in terms of light spectrum. Lighting Research \& Technology, 45(6), 641-665. (DOI: 10.1177/1477153513475913)

JUDD, D.B. 1967. The flattery index for Artificial Illuminants. Illuminating Engineering, 62(10), 593-598.

NEWHALL, S.M., BURNHAM, R.W. and CLARK, J.R. 1957. Comparison of Successive with Simultaneous Colour Matching, Journal of the Optical Society of America, 47(1), 43-56. (DOI: 10.1364/JOSA.47.000043)

SANDERS, C.L. 1959. Colour Preference for Naural Objects. Illuminating Engineering, 54(7), 452-456.

WEI, M., BAO, W. and HUANG, H.-P. 2018. Consideration of Light Level in Specifying Light Source Colour Rendition. LEUKOS, 1-11. (DOI: 10.1080/15502724.2018.1448992) 